\title{
On Noncrossing and Plane Tree-Like Structures
}

\author{
Isaac Owino Okoth ${ }^{1 *}$
}

\begin{abstract}
Mathematical trees are connected graphs without cycles, loops and multiple edges. Various trees such as Cayley trees, plane trees, binary trees, $d$-ary trees, noncrossing trees among others have been studied extensively. Tree-like structures such as Husimi graphs and cacti are graphs which posses the conditions for trees if, instead of vertices, we consider their blocks. In this paper, we use generating functions and bijections to find formulas for the number of noncrossing Husimi graphs, noncrossing cacti and noncrossing oriented cacti. We extend the work to obtain formulas for the number of bicoloured noncrossing Husimi graphs, bicoloured noncrossing cacti and bicoloured noncrossing oriented cacti. Finally, we enumerate plane Husimi graphs, plane cacti and plane oriented cacti according to number of blocks, block types and leaves.

Keywords: Noncrossing tree, Plane tree, Tree-like structure, Husimi graph, Cactus, Oriented cactus, Enumeration

2010 AMS: Primary 05C30, Secondary 05C05, 05A19
\end{abstract}

${ }^{1}$ Department of Pure and Applied Mathematics, Maseno University, Maseno, Kenya, ORCID: 0000-0003-4503-4733

*Corresponding author: ookoth@maseno.ac.ke

Received: 30 September 2020, Accepted: 9 June 2021, Available online: 30 June 2021

\section{Introduction}

Husimi graph is a connected graph whose blocks are complete graphs. These graphs were introduced and enumerated by Japanese physicist Kodi Husimi in [5]. If the blocks of a connected graph are polygons then the graph is called a cactus. Cacti were introduced by Harary and Uhlenbeck in [4] where they appeared as Husimi trees. In 1996, Collin Springer [12] introduced and enumerated oriented cacti. These are connected graphs whose blocks are oriented cycles. Formulas counting these tree-like structures as well as their coloured counterparts, i.e. structures coloured with the property that blocks of the same colour are not incident to one another, have been obtained. See $[1,3-5,7,8,10,12]$ for details. In this paper, we enumerate their noncrossing and plane counterparts. The degree of a vertex in a tree-like structure is the number of blocks that are incident to it.

This paper is organized as follows: In Section 2, we enumerate noncrossing Husimi graphs, cacti and oriented cacti by block type and number of blocks. A bijection between these structures and certain polygon dissections is also presented here. Noncrossing tree-like structures whose blocks are coloured using two colours such that no blocks of the same colour are incident to one another are enumerated in Section 3. Lastly in Section 4, we enumerate plane tree-like structures according to block sizes, block types and number of leaves. Some of the results presented here were part of the author's PhD thesis [10].

\section{Noncrossing tree-like structures}

In this section, we obtain equivalent results for Husimi graphs, cacti and oriented cacti whose blocks do not cross. We shall call these structures as noncrossing Husimi graphs, noncrossing cacti and noncrossing oriented cacti respectively. The simplest of the noncrossing structures is a noncrossing tree. This is a tree drawn in the plane with vertices on the boundary of a circle such that the edges do not cross inside the circle. Marc Noy [9] showed that the number of noncrossing trees on $n$ labelled vertices is 
given by

$$
\frac{1}{2 n-1}\left(\begin{array}{c}
3 n-3 \\
n-1
\end{array}\right)
$$

This result was later generalised to connected graphs by Flajolet and Noy [2]. Before we embark on the enumeration of noncrossing Husimi graphs, let us review the notion of butterfly decomposition of noncrossing trees that was introduced in [2]. A butterfly is an ordered pair of trees that share a root. If a vertex $v$ in a tree has degree $d$, then the tree can be decomposed into $d$ butterflies hanging from $v$.

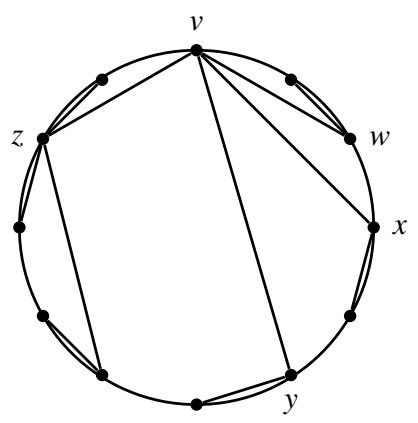

Figure 2.1. Noncrossing tree

In Figure 2.1, there are 4 butterflies rooted at $w, x, y$ and $z$. The aforementioned authors showed that if $T(x)$ is the generating function for trees and $B(x)$ is the generating function for butterflies then we have the following equations:

$$
T(x)=\frac{x}{1-B} \text { and } B(x)=\frac{T^{2}}{x} .
$$

Theorem 2.1. Let $\left(n_{2}, n_{3}, \ldots\right)$ be a sequence of non-negative integers satisfying the condition that $n=\sum_{j \geq 2}(j-1) n_{j}+1$. The number $\mathrm{NHG}_{n}\left(n_{2}, n_{3}, \ldots\right)$ of noncrossing Husimi graphs on $[n]$ having $n_{j}$ blocks of size $j$ is given by

$$
N H G_{n}\left(n_{2}, n_{3}, \ldots\right)=\frac{(2 n+k-2) !}{(2 n-1) ! \prod_{j \geq 2} n_{j} !}
$$

where $k=\sum_{j \geq 2} n_{j}$.

Proof. Let $F(x)$ be the generating function for noncrossing Husimi graphs. Let $y_{i}$ mark the number of vertices in each block. Adopting the butterfly decomposition of noncrossing trees to noncrossing Husimi graphs, we have that

$$
F(x)=\frac{x}{1-\sum_{i \geq 1} y_{i+1} B^{i}}
$$

and

$$
B(x)=\frac{F^{2}}{x}
$$

where $B(x)$ is the generating function for butterflies.

Therefore the generating function $F(x)$ satisfies

$$
F(x)=\frac{x}{1-\sum_{i \geq 1} y_{i+1}\left(\frac{F^{2}}{x}\right)^{i}} .
$$

Thus for $G=\frac{F}{\sqrt{x}}$ we have

$$
G(x)=\frac{\sqrt{x}}{1-\sum_{i \geq 1} y_{i+1} G^{2 i}} .
$$


By the Lagrange Inversion Formula, we obtain

$$
\begin{aligned}
{\left[x^{n}\right] F(x) } & =\left[x^{n-\frac{1}{2}}\right] G(x)=\frac{1}{2 n-1}\left[t^{2 n-2}\right]\left(1-\sum_{i \geq 1} y_{i+1} t^{2 i}\right)^{-(2 n-1)} \\
& =\frac{1}{2 n-1}\left[t^{2 n-2}\right] \sum_{k \geq 0}\left(\begin{array}{c}
-(2 n-1) \\
k
\end{array}\right)\left(-\sum_{i \geq 1} y_{i+1} t^{2 i}\right)^{k} \\
& =\frac{1}{2 n-1}\left[t^{2 n-2}\right] \sum_{k \geq 0}\left(\begin{array}{c}
2 n+k-2 \\
k
\end{array}\right)\left(\sum_{i \geq 1} y_{i+1} t^{2 i}\right)^{k} \\
& =\frac{1}{2 n-1} \sum_{k \geq 0}\left(\begin{array}{c}
2 n+k-2 \\
k
\end{array}\right) \sum_{\substack{n_{2}+n_{3}+\cdots=k \\
n_{2}+2 n_{3}+\cdots=n-1}} \frac{k y_{2}^{n_{2}} y_{3}^{n_{3}} \cdots}{n_{2} ! n_{3} ! \cdots} .
\end{aligned}
$$

Therefore,

$$
N H G_{n}\left(n_{2}, n_{3}, \ldots\right)=\frac{1}{2 n-1}\left(\begin{array}{c}
2 n+k-2 \\
k
\end{array}\right) \frac{k !}{\prod_{j \geq 2} n_{j} !} .
$$

Corollary 2.2. The number of noncrossing Husimi graphs on $n \geq 2$ vertices is given by

$$
\frac{1}{n-1} \sum_{k=1}^{n-1}\left(\begin{array}{c}
2 n+k-2 \\
k-1
\end{array}\right)\left(\begin{array}{c}
n-1 \\
k
\end{array}\right)
$$

Proof. We need to show that the number of noncrossing Husimi graphs on $n$ vertices with $k$ blocks is given by the generalised Narayana number,

$$
\frac{1}{n-1}\left(\begin{array}{c}
2 n+k-2 \\
k-1
\end{array}\right)\left(\begin{array}{c}
n-1 \\
k
\end{array}\right)
$$

Let $[[n, k]]$ denote the set of all types of partitions of $[n]$ of length $k$. Since

$$
\sum_{P \in[[n-1, k]]} \frac{k !}{n_{2} ! n_{3} ! \cdots}=\left(\begin{array}{l}
n-2 \\
k-1
\end{array}\right),
$$

the result follows from Equation (2.2).

The formula (2.3) appears in [11] and [14] as the number of dissections of a convex polygon on $2 n$ vertices with $k-1$ noncrossing diagonals such that the number of edges enclosing each interior region is even. We now construct a bijection between the set of these dissections and the noncrossing Husimi graphs.

Lemma 2.3. There is a bijection between the set of dissections of a convex polygon on $2 n$ vertices with $k-1$ noncrossing diagonals such that the number of edges enclosing each interior region is divisible by two and the set of noncrossing Husimi graphs on $n$ vertices with $k$ blocks.

Proof. Consider a convex polygon on $2 n$ vertices such that the vertices are labelled in clockwise direction as $1,1^{\prime}, 2,2^{\prime}, \ldots, n, n^{\prime}$. Let the number of noncrossing diagonal edges be $k-1$ and the number of edges of each interior region be divisible by 2 . There are $k$ such regions. Create an edge between any two vertices of label $1,2, \ldots, n$ that are in the same region. A vertex which is incident to more than one region is considered to belong to all the incident regions. The resultant graph is a noncrossing Husimi graph on $n$ vertices with $k$ blocks. See Figure 2.2 for an example. The process can easily be reversed. 

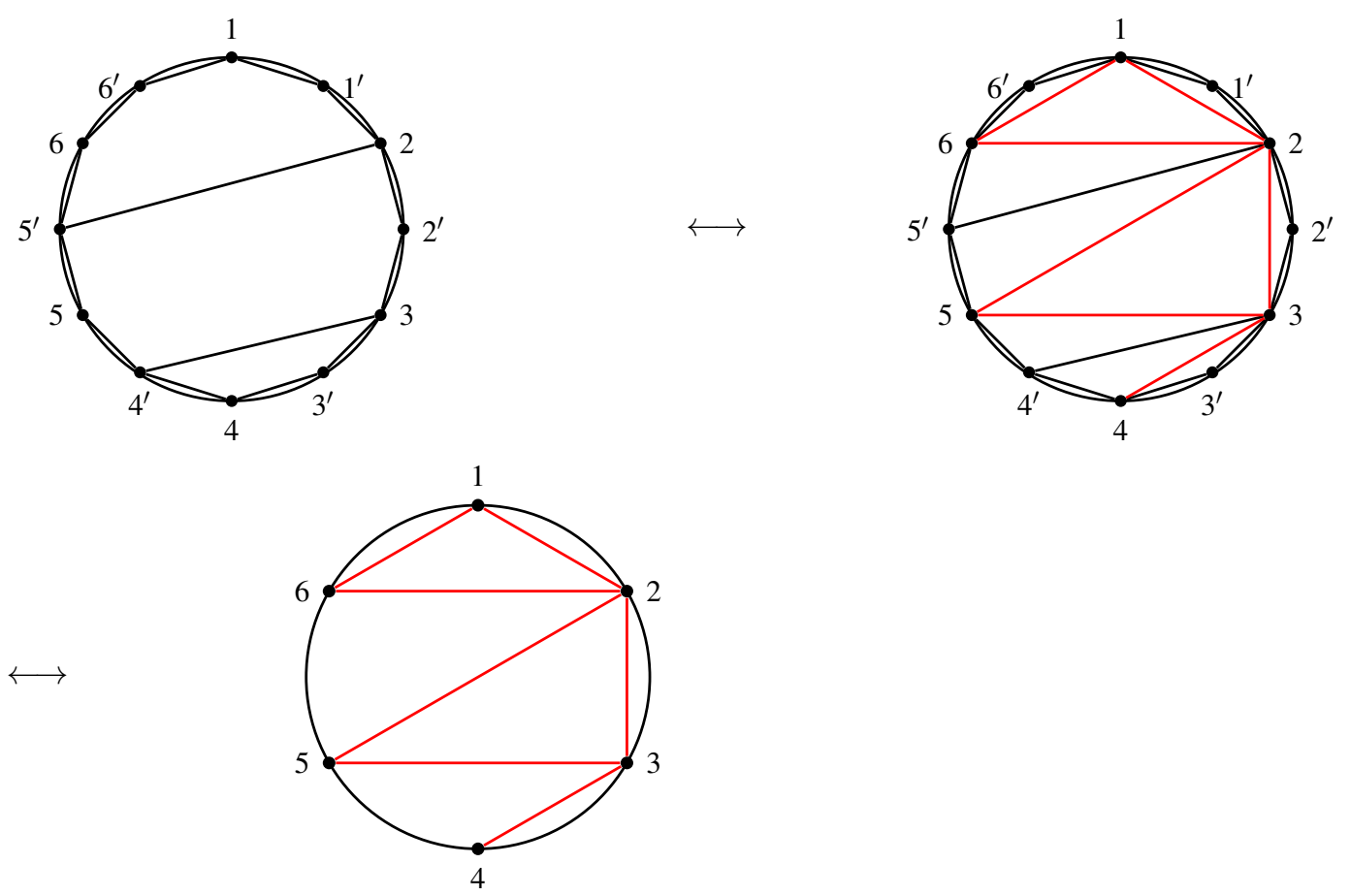

Figure 2.2. Diagram showing the bijection in the proof of Lemma 2.3.

We obtain further corollaries of Theorem 2.1.

Corollary 2.4. Let $\left(n_{2}, n_{3}, \ldots\right)$ be a sequence of non-negative integers satisfying the condition that $n=\sum_{j \geq 2}(j-1) n_{j}+1$. The number $\mathrm{NC}_{n}\left(n_{2}, n_{3}, \ldots\right)$ of noncrossing cacti on $[n]$ having $n_{j}$ blocks of size $j$ is given by

$$
\mathrm{NC}_{n}\left(n_{2}, n_{3}, \ldots\right)=\frac{(2 n+k-2) !}{(2 n-1) ! \prod_{j \geq 2} n_{j} !}
$$

where $k=\sum_{j \geq 2} n_{j}$.

Proof. In the noncrossing setting, there is only one way to turn a complete graph into a cycle thus the required equation follows from Equation (2.1) i.e.,

$$
N C_{n}\left(n_{2}, n_{3}, \ldots\right)=N H G_{n}\left(n_{2}, n_{3}, \ldots\right) \text {. }
$$

Corollary 2.5. The number of noncrossing cacti on $[n]$, where $n \geq 2$, is

$$
\frac{1}{n-1} \sum_{k=1}^{n-1}\left(\begin{array}{c}
2 n+k-2 \\
k-1
\end{array}\right)\left(\begin{array}{c}
n-1 \\
k
\end{array}\right)
$$

Proof. We obtain the formula by summing over all possibilities of $n_{2}, n_{3}, \ldots$ and $k$ as in the proof of Corollary 2.2.

Corollary 2.6. Let $\left(n_{2}, n_{3}, \ldots\right)$ be a sequence of non-negative integers satisfying the condition that $n=\sum_{j \geq 2}(j-1) n_{j}+1$. The number $\mathrm{NOC}_{n}\left(n_{2}, n_{3}, \ldots\right)$ of noncrossing oriented cacti on $[n]$ having $n_{j}$ blocks of size $j$ is given by

$$
\operatorname{NOC}_{n}\left(n_{2}, n_{3}, \ldots\right)=\frac{(2 n+k-2) ! 2^{k-n_{2}}}{(2 n-1) ! \prod_{j \geq 2} n_{j} !},
$$

where $k=\sum_{j \geq 2} n_{j}$.

Proof. Since any polygon of size $\geq 3$ has 2 orientations, we have

$$
N O C_{n}\left(n_{2}, n_{3}, \ldots\right)=2^{k-n_{2}} \cdot N C_{n}\left(n_{2}, n_{3}, \ldots\right) .
$$

The formula thus follows from Equation (2.4). 
Corollary 2.7. The number of noncrossing oriented cacti on $[n]$, where $n \geq 2$, is

$$
\sum_{k \geq 0} \sum_{\begin{array}{c}
n_{2}+n_{3}+\cdots=k \\
n_{2}+2 n_{3}+\cdots=n-1
\end{array}} \frac{(2 n+k-2) ! 2^{k-n_{2}}}{(2 n-1) ! \prod_{j \geq 2} n_{j} !} .
$$

\section{Bicoloured noncrossing tree-like structures}

In the next proposition, we obtain a formula for the number of noncrossing Husimi graphs on $n$ labelled vertices such that the degrees of the vertices are less than or equal to 2. This will make 2-colouring possible. Recall, from Section 1, that the degree of a vertex $v$ in a Husimi graph is the number of blocks that are incident to it.

Proposition 3.1. Let $\mathrm{NHG}_{n, 2}\left(n_{2}, n_{3}, \ldots\right)$ be the number of noncrossing Husimi graphs on $[n]$ having $n_{i}$ blocks of size $i$ such that $\sum_{i \geq 2}(i-1) n_{i}+1=n$ and all the vertices have degree less than or equal to 2 . Then

$$
N H G_{n, 2}\left(n_{2}, n_{3}, \ldots\right)=\frac{n ! 2^{k-1}}{(n-k+1) ! \prod_{j \geq 2} n_{j} !}
$$

where $k=\sum_{j \geq 2} n_{j}$.

Proof. Let $F(x)$ be the generating function for 2-colourable noncrossing Husimi graphs with root degree 1 (or 0). Let $y_{i}$ mark blocks of size $i$. Since each vertex in the block is to have degree less than or equal to two, the generating function satisfies

$$
F(x)=x\left(1+\sum_{i \geq 1} y_{i+1}(2 F-x)^{i}\right) .
$$

The butterflies of these graphs must be rooted at vertices of degree 1 (or consists of a single vertex). We subtract $x$ to cater for cases in which a butterfly consists of a single vertex.

Setting $G=2 F-x$ in Equation (3.2) we obtain

$$
G=x\left(1+2 \sum_{i \geq 1} y_{i+1} G^{i}\right) .
$$

$G$ is the generating function for 2-coloured Husimi graphs with root degree 1 (in the case of a single vertex, there are no blocks, thus nothing to be coloured; otherwise there are precisely two colourings). When $y_{2}=y_{3}=\cdots=1$, then we obtain the generating function for the large Schröder numbers.

Now, for arbitrary root degree, root degree 2 Husimi graphs are obtained by merging two root degree 1 Husimi graphs. We subtract $F$ for double counting root degree 1 Husimi graphs. The generating function is thus

$$
H(x)=\frac{F^{2}}{x}-F=\frac{G^{2}}{4 x}-\frac{x}{4} .
$$

This implies that

$$
\left[x^{n}\right] H=\frac{1}{4}\left[x^{n+1}\right] G^{2} .
$$

By the Lagrange Inversion Formula, we have

$$
\begin{aligned}
\frac{1}{4}\left[x^{n+1}\right] G^{2} & =\frac{1}{2(n+1)}\left[t^{n-1}\right]\left(1+2 \sum_{i \geq 1} y_{i+1} t^{i}\right)^{n+1} \\
& =\frac{1}{2(n+1)}\left[t^{n-1}\right] \sum_{k \geq 0}\left(\begin{array}{c}
n+1 \\
k
\end{array}\right)\left(2 \sum_{i \geq 1} y_{i+1} t^{i}\right)^{k} \\
& =\frac{1}{2(n+1)} \sum_{k \geq 0} 2^{k}\left(\begin{array}{c}
n+1 \\
k
\end{array}\right) \sum_{\begin{array}{c}
n_{2}+n_{3}+\cdots=k \\
n_{2}+2 n_{3}+\cdots=n-1
\end{array}} \frac{k ! y_{2}^{n_{2}} y_{3}^{n_{3}} \cdots}{n_{2} ! n_{3} ! \cdots}
\end{aligned}
$$

Therefore,

$$
N H G_{n, 2}\left(n_{2}, n_{3}, \ldots\right)=\frac{2^{k-1}}{n+1}\left(\begin{array}{c}
n+1 \\
k
\end{array}\right) \cdot \frac{k !}{n_{2} ! n_{3} ! \cdots} .
$$


Corollary 3.2. There are $n \cdot 2^{n-3}$ noncrossing trees on $n \geq 2$ vertices such that all the vertices have degree less than or equal to 2 .

Proof. The result follows from Equation (3.3) by taking $\left(n_{2}, n_{3}, \ldots\right)=(n-1,0, \ldots)$ so that $k=n-1$.

Observe that these trees are also noncrossing paths. The corollary thus follows by a simple counting argument as well: first choose a root (in $n$ ways), then 2 choices for each step.

Corollary 3.3. Let $N H G_{n, 2}$ be the number of noncrossing Husimi graphs on $[n]$ in which all the vertices have degree at most 2 . Then

$$
N H G_{n, 2}=\frac{1}{n-1} \sum_{k=1}^{n-1} 2^{k-1}\left(\begin{array}{c}
n \\
k-1
\end{array}\right)\left(\begin{array}{c}
n-1 \\
k
\end{array}\right) .
$$

Proof. To prove Formula (3.4), we need to show that the number of noncrossing Husimi graphs on $n$ vertices with $k$ blocks in which each vertex has degree $\leq 2$ is given by

$$
\frac{2^{k-1}}{n-1}\left(\begin{array}{c}
n \\
k-1
\end{array}\right)\left(\begin{array}{c}
n-1 \\
k
\end{array}\right)
$$

Since

$$
\sum_{P \in[n-1, k]]} \frac{k !}{n_{2} ! n_{3} ! \cdots}=\left(\begin{array}{l}
n-2 \\
k-1
\end{array}\right),
$$

the result follows from Equation (3.3).

Lemma 3.4. The number of bicoloured noncrossing Husimi graphs on $[n]$ having $n_{i}$ blocks of size $i$ such that $\sum_{i \geq 2}(i-1) n_{i}+1=$ $n$ is equal to

$$
\frac{n ! 2^{k}}{(n-k+1) ! \prod_{j \geq 2} n_{j} !}
$$

where $k=\sum_{j \geq 2} n_{j}$.

Proof. Consider a noncrossing Husimi graph on $[n]$ having $n_{i}$ blocks of size $i$ such that $\sum_{i \geq 2}(i-1) n_{i}+1=n$ and with vertices having degree less than or equal to 2 . Let $b$ be a block in the graph. There are two choices for colouring block $b$ and one choice for the remaining blocks. The result thus follows from Equation (3.1).

Corollary 3.5. The number of bicoloured noncrossing Husimi graphs on $n$ vertices is given by

$$
\frac{1}{n-1} \sum_{k=1}^{n-1} 2^{k}\left(\begin{array}{c}
n \\
k-1
\end{array}\right)\left(\begin{array}{c}
n-1 \\
k
\end{array}\right) .
$$

We obtain the following special case by setting $k=n-1$ in Equation (3.5).

Corollary 3.6. There are $n \cdot 2^{n-2}$ bicoloured noncrossing trees on $n \geq 2$ labelled vertices.

Corollary 3.7. The number of bicoloured noncrossing cacti on $[n]$ having $n_{i}$ cycles of size $i$ such that $\sum_{i \geq 2}(i-1) n_{i}+1=n$ is equal to

$$
\frac{n ! 2^{k}}{(n-k+1) ! \prod_{j \geq 2} n_{j} !},
$$

where $k=\sum_{j \geq 2} n_{j}$.

Corollary 3.8. The number of bicoloured noncrossing cacti on $[n]$, where $n \geq 2$, is

$$
\frac{1}{n-1} \sum_{k=1}^{n-1} 2^{k}\left(\begin{array}{c}
n \\
k-1
\end{array}\right)\left(\begin{array}{c}
n-1 \\
k
\end{array}\right)
$$


Corollary 3.9. The number of bicoloured noncrossing oriented cacti on $[n]$ having $n_{i}$ cycles of size $i$ such that $\sum_{i \geq 2}(i-1) n_{i}+1=$ $n$ is equal to

$$
\frac{n ! 2^{2 k-n_{2}}}{(n-k+1) ! \prod_{j \geq 2} n_{j} !},
$$

where $k=\sum_{j \geq 2} n_{j}$.

Corollary 3.10. The number of bicoloured noncrossing oriented cacti on $[n]$, for $n \geq 2$, is

$$
\sum_{k \geq 0} \sum_{\substack{n_{2}+n_{3}+\cdots=k \\ n_{2}+2 n_{3}+\cdots=n-1}} \frac{n ! 2^{2 k-n_{2}}}{(n-k+1) ! \prod_{j \geq 2} n_{j} !} .
$$

\section{Plane tree-like structures}

A plane Husimi graph (resp. plane cactus) is a Husimi graph (resp. cactus) drawn on the plane such that its blocks are ordered (see, Figure 4.1 for plane cactus).

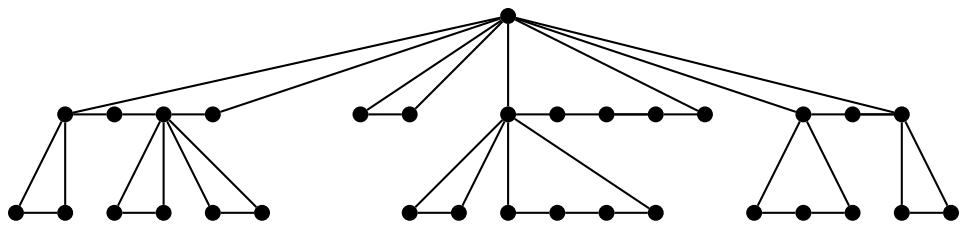

Figure 4.1. Plane cactus on 32 vertices.

In this section, we shall call the number of blocks coming out of a vertex as the degree of that vertex. A leaf is a non-root vertex which is incident to exactly one block. A non-leaf vertex is referred to as internal vertex.

Theorem 4.1. Let $\left(n_{2}, n_{3}, \ldots\right)$ be a sequence of non-negative integers satisfying the coherence condition: $n=\sum_{j \geq 2}(j-1) n_{j}+1$. The number $\mathrm{PHG}_{n}\left(n_{2}, n_{3}, \ldots\right)$ of plane Husimi graphs on $n$ vertices having $n_{j}$ blocks of size $j$ is given by

$$
P H G_{n}\left(n_{2}, n_{3}, \ldots\right)=\frac{(n+k-1) !}{n ! \prod_{j \geq 2} n_{j} !}
$$

where $k=\sum_{j \geq 2} n_{j}$.

Proof. Let $P(x)$ be the generating function for plane Husimi graphs. Let $y_{i}$ mark the number of vertices in each block. Then we have

$$
P(x)=\frac{x}{1-\sum_{i \geq 1} y_{i+1} P^{i}} .
$$

By the Lagrange Inversion Formula [13], we obtain

$$
\begin{aligned}
{\left[x^{n}\right] P(x) } & =\frac{1}{n}\left[t^{n-1}\right]\left(1-\sum_{i \geq 1} y_{i+1} t^{i}\right)^{-n} \\
& =\frac{1}{n}\left[t^{n-1}\right] \sum_{k \geq 0}\left(\begin{array}{c}
-n \\
k
\end{array}\right)\left(-\sum_{i \geq 1} y_{i+1} t^{t}\right)^{k} \\
& =\frac{1}{n}\left[t^{n-1}\right] \sum_{k \geq 0}\left(\begin{array}{c}
n+k-1 \\
k
\end{array}\right)\left(\sum_{i \geq 1} y_{i+1} t^{i}\right)^{k} \\
& =\frac{1}{n} \sum_{k \geq 0}\left(\begin{array}{c}
n+k-1 \\
k
\end{array}\right) \sum_{\substack{n_{2}+n_{3}+\cdots=k \\
n_{2}+2 n_{3}+\cdots=n-1}} \frac{k ! y_{2}^{n_{2}} y_{3}^{n_{3}} \cdots}{n_{2} ! n_{3} ! \cdots} .
\end{aligned}
$$


Therefore,

$$
P H G_{n}\left(n_{2}, n_{3}, \ldots\right)=\frac{1}{n}\left(\begin{array}{c}
n+k-1 \\
k
\end{array}\right) \frac{k !}{\prod_{j \geq 2} n_{j} !} .
$$

This completes the proof.

In the proof of the following corollary, we get a formula for the number of plane Husimi graphs with a given number of blocks.

Corollary 4.2. The number of plane Husimi graphs on $n \geq 2$ vertices is given by

$$
\frac{1}{n} \sum_{k=1}^{n-1}\left(\begin{array}{c}
n+k-1 \\
k
\end{array}\right)\left(\begin{array}{l}
n-2 \\
k-1
\end{array}\right)
$$

Proof. We need to show that the number of plane Husimi graphs on $n$ vertices with $k$ blocks is given by,

$$
\frac{1}{n}\left(\begin{array}{c}
n+k-1 \\
k
\end{array}\right)\left(\begin{array}{l}
n-2 \\
k-1
\end{array}\right) \text {. }
$$

Let $P(n, k)$ denote the set of all types of partitions of $\{1,2, \ldots, n\}$ of length $k$. Since

$$
\sum_{P \in P(n-1, k)} \frac{k !}{n_{2} ! n_{3} ! \cdots}=\left(\begin{array}{c}
n-2 \\
k-1
\end{array}\right)
$$

then the formula follows from Equation (4.2).

Setting $k=n-1$ in Equation (4.3), we recover the formula for plane trees on $n$ vertices. Similarly, setting $n=d n+1$, $n_{d+1}=n$ and $n_{i}=0$ for all $i \neq d+1$, in Equation (4.1), we rediscover the formula

$$
\frac{1}{d n+1}\left(\begin{array}{c}
(d+1) n \\
n
\end{array}\right)
$$

for the number of $d$-tuplet trees on $d n+1$ vertices obtained in [6]. Here, if $d=1$ we get the number of plane trees on $n+1$ vertices.

Corollary 4.3. Let $\left(n_{2}, n_{3}, \ldots\right)$ be a sequence of non-negative integers satisfying the condition that $n=\sum_{j \geq 2}(j-1) n_{j}+1$. The number $\mathrm{PC}_{n}\left(n_{2}, n_{3}, \ldots\right)$ of plane cacti on $n$ nodes and having $n_{j}$ blocks of size $j$ is given by

$$
\mathrm{PC}_{n}\left(n_{2}, n_{3}, \ldots\right)=\frac{(n+k-1) !}{n ! \prod_{j \geq 2} n_{j} !},
$$

where $k=\sum_{j \geq 2} n_{j}$.

Proof. Since there is only one way to turn a complete graph into a cycle, the required equation follows from Equation (4.1) i.e.,

$$
P C_{n}\left(n_{2}, n_{3}, \ldots\right)=P H G_{n}\left(n_{2}, n_{3}, \ldots\right) .
$$

Corollary 4.4. The number of plane cacti on $n$ nodes, where $n \geq 2$, is

$$
\frac{1}{n} \sum_{k=1}^{n-1}\left(\begin{array}{c}
n+k-1 \\
k
\end{array}\right)\left(\begin{array}{l}
n-2 \\
k-1
\end{array}\right) .
$$

Proof. We obtain the formula by summing over all possibilities of $n_{2}, n_{3}, \ldots$ and $k$ as in the proof of Corollary 4.2.

Corollary 4.5. Let $\left(n_{2}, n_{3}, \ldots\right)$ be a sequence of non-negative integers satisfying the condition that $n=\sum_{j \geq 2}(j-1) n_{j}+1$. The number $\mathrm{POC}_{n}\left(n_{2}, n_{3}, \ldots\right)$ of plane oriented cacti on $n$ vertices and having $n_{j}$ blocks of size $j$ is given by

$$
\operatorname{POC}_{n}\left(n_{2}, n_{3}, \ldots\right)=\frac{(n+k-1) ! 2^{k-n_{2}}}{n ! \prod_{j \geq 2} n_{j} !},
$$

where $k=\sum_{j \geq 2} n_{j}$. 
Proof. Since any polygon of size $\geq 3$ has 2 orientations, we have

$$
\operatorname{POC}_{n}\left(n_{2}, n_{3}, \ldots\right)=2^{k-n_{2}} \cdot P C_{n}\left(n_{2}, n_{3}, \ldots\right) .
$$

The result follows from Equation (4.5).

Corollary 4.6. The number of plane oriented cacti on $n$ vertices, where $n \geq 2$, is

$$
\sum_{k \geq 0} \sum_{\substack{n_{2}+n_{3}+\cdots=k \\ n_{2}+2 n_{3}+\cdots=n-1}} \frac{(n+k-1) ! 2^{k-n_{2}}}{n ! \prod_{j \geq 2} n_{j} !} .
$$

For the rest of this paper, we are interested in the number of plane tree-like structures with a given number of leaves.

Theorem 4.7. Let $\left(n_{2}, n_{3}, \ldots\right)$ be a sequence of non-negative integers satisfying the coherence condition: $n=\sum_{j \geq 2}(j-1) n_{j}+1$. The number of plane Husimi graphs on $n$ vertices with $\ell$ leaves and having $n_{j}$ blocks of size $j$ is given by

$$
\frac{1}{n}\left(\begin{array}{c}
n \\
\ell
\end{array}\right)\left(\begin{array}{c}
k-1 \\
n-\ell-1
\end{array}\right) \frac{k !}{\prod_{j \geq 2} n_{j} !}
$$

where $k=\sum_{j \geq 2} n_{j}$.

Proof. Let $F(x, u)$ be the bivariate generating function for the number of plane Husimi graphs such that $x$ and $u$ are marking vertices and leaves respectively. Again $y_{i}$ will mark the number of vertices in each block.

Now,

$$
F(x, u)=x u+\frac{x}{1-\sum_{i \geq 1} y_{i+1} F(x, u)^{i}}-x .
$$

For convenience, let $w=F(x, u)$ so that $w=x\left(u+\frac{\sum_{i \geq 1} y_{i+1} w^{i}}{1-\sum_{i \geq 1} y_{i+1} w^{i}}\right)$. We extract the coefficients of $x^{n}$ and $u^{\ell}$ in the generating function.

$$
\begin{aligned}
{\left[x^{n} u^{\ell}\right] F(x, u)=\left[x^{n} u^{\ell}\right] w } & =\frac{1}{n}\left[u^{\ell} t^{n-1}\right]\left(u+\frac{\sum_{i \geq 1} y_{i+1} t^{i}}{1-\sum_{i \geq 1} y_{i+1} t^{i}}\right)^{n} \\
& =\frac{1}{n}\left[u^{\ell} t^{n-1}\right] \sum_{j=0}^{n}\left(\begin{array}{c}
n \\
j
\end{array}\right) u^{j}\left(\frac{\sum_{i \geq 1} y_{i+1} t^{i}}{1-\sum_{i \geq 1} y_{i+1} t^{i}}\right)^{n-j} \\
& =\frac{1}{n}\left(\begin{array}{c}
n \\
\ell
\end{array}\right)\left[t^{n-1}\right]\left(\frac{\sum_{i \geq 1} y_{i+1} t^{i}}{1-\sum_{i \geq 1} y_{i+1} t^{i}}\right)^{n-\ell} \\
& =\frac{1}{n}\left(\begin{array}{c}
n \\
\ell
\end{array}\right)\left[t^{n-1}\right]\left(\sum_{i \geq 1} y_{i+1} t^{i}\right)^{n-\ell}\left(1-\sum_{i \geq 1} y_{i+1} t^{i}\right)^{-(n-\ell)} \\
& =\frac{1}{n}\left(\begin{array}{c}
n \\
\ell
\end{array}\right)\left[t^{n-1}\right] \sum_{j=0}^{n-\ell}\left(\begin{array}{c}
-(n-\ell) \\
j
\end{array}\right)\left(-\sum_{i \geq 1} y_{i+1} t^{i}\right)^{j}\left(\sum_{i \geq 1} y_{i+1} t^{i}\right)^{n-\ell} \\
& =\frac{1}{n}\left(\begin{array}{c}
n \\
\ell
\end{array}\right)\left[t^{n-1}\right] \sum_{j=0}^{n-\ell}\left(\begin{array}{c}
n-\ell+j-1 \\
j
\end{array}\right)\left(\sum_{i \geq 1} y_{i+1} t^{i}\right)^{n-\ell+j} .
\end{aligned}
$$

Let $k=n-\ell+j$ so that

$$
\left[x^{n} u^{\ell}\right] F(x, u)=\frac{1}{n}\left(\begin{array}{c}
n \\
\ell
\end{array}\right) \sum_{k=n-\ell}^{2 n-2 \ell}\left(\begin{array}{c}
k-1 \\
n-\ell-1
\end{array}\right) \sum_{\substack{n_{2}+n_{3}+\cdots=k \\
n_{2}+2 n_{3}+\cdots=n-1}} \frac{k ! y_{2}^{n_{2}} y_{3}^{n_{3}} \cdots}{n_{2} ! n_{3} ! \cdots} .
$$

This completes the proof. 
From Equation (4.6) and summing over all $n_{j}$ as in Equation (4.4), it follows that there are

$$
\frac{1}{n}\left(\begin{array}{c}
n \\
\ell
\end{array}\right)\left(\begin{array}{c}
k-1 \\
n-\ell-1
\end{array}\right)\left(\begin{array}{l}
n-2 \\
k-1
\end{array}\right)
$$

plane Husimi graphs on $n$ vertices with $k$ blocks and having exactly $\ell$ leaves. Setting $k=n-1$, we rediscover the famous Narayana number for the number of plane trees with a given number of leaves. Summing over all $\ell$, making use of Vandermonde convolution, we obtain Equation (4.3) for the number of plane Husimi graphs on $n$ vertices.

The expected number of leaves in plane Husimi graphs on $n$ vertices with $k$ blocks is

$$
\sum_{\ell=1}^{n-1} \frac{\ell}{n}\left(\begin{array}{c}
n \\
\ell
\end{array}\right)\left(\begin{array}{c}
k-1 \\
n-\ell-1
\end{array}\right)\left(\begin{array}{l}
n-2 \\
k-1
\end{array}\right)=\left(\begin{array}{c}
n+k-2 \\
k
\end{array}\right)\left(\begin{array}{l}
n-2 \\
k-1
\end{array}\right)
$$

and upon division by Equation (4.3), we get that on average there are $\left(n^{2}-n\right) /(n+k-1)$ leaves in the aforementioned plane graphs.

Setting $r=n-\ell$ in Equation (4.7), we obtain the following result.

Corollary 4.8. There are

$$
\frac{1}{n}\left(\begin{array}{l}
n \\
r
\end{array}\right)\left(\begin{array}{l}
k-1 \\
r-1
\end{array}\right)\left(\begin{array}{l}
n-2 \\
k-1
\end{array}\right)
$$

plane Husimi graphs on $n$ vertices with $k$ blocks and having $r$ internal vertices.

\section{Acknowledgements}

The authors would like to express their sincere thanks to the editor and the anonymous reviewers for their helpful comments and suggestions.

\section{Funding}

There is no funding for this work.

\section{Availability of data and materials}

Not applicable.

\section{Competing interests}

The authors declare that they have no competing interests.

\section{Author's contributions}

All authors contributed equally to the writing of this paper. All authors read and approved the final manuscript.

\section{References}

[1] M. Bóna, M. Bousquet, G. Labelle, P. Leroux, Enumeration of m-ary cacti, Adv. Appl. Math., 24 (1) (2000), $22-56$.

[2] P. Flajolet, M. Noy, Analytic combinatorics of non-crossing configurations, Discrete Math., 204 (1-3) (1999), $203-229$.

[3] G. W. Ford, G. E. Uhlenbeck, Combinatorial Problems in the Theory of Graphs, I, Proc. Nat. Acad. Sciences, 42 (1956), 122-128.

[4] F. Harary, G. E. Uhlenbeck, On the number of Husimi trees, Proc. Nat. Aca. Sci., 39 (1953), $315-322$.

[5] K. Husimi, Note on Mayers' theory of cluster integrals, J. Chem. Phys., 18 (1950), 682-684.

[6] S. Kim, S. Seo, H. Shin, Refined enumeration of vertices among all rooted rooted d-trees, (2018), arXiv:1806.06417.

[7] P. Leroux. Enumerative problems inspired by Mayer's theory of cluster integrals, Electron. J. Combin., 11 (2004). 
[8] J. E. Mayer, Equilibrium Statistical Mechanics, The international encyclopedia of physical chemistry and chemical physics, Pergamon Press, Oxford, 1968.

[9] M. Noy, Enumeration of noncrossing trees on a circle, Discrete Math., 180 (1-3) (1998), 301-313.

[10] I. O. Okoth, Combinatorics of oriented trees and tree-like structures, PhD Thesis, Stellenbosch University, 2015.

[11] J. H. Przytycki, A. S. Sikora, Polygon Dissections and Euler, Fuss, Kirkman, and Cayley Numbers, J. Combin. Theory, Ser. A, 92 (1) (2000), 68-76.

[12] C. Springer, Factorizations, Trees, and Cacti, Proceedings of the Eighth International Conference on Formal Power Series and Algebraic Combinatorics (FPSAC), University of Minnesota,(1996), 427-438.

${ }^{[13]}$ R. P. Stanley. Enumerative Combinatorics. Vol. 2, volume 62 of Cambridge Studies in Advanced Mathematics. Cambridge University Press, Cambridge, 1999.

[14] E. Tzanaki, Polygon dissections and some generalizations of cluster complexes, J. Combin. Theory, Ser. A, 113(6) (2006), 1189-1198. 